\title{
DISTRIBUTION OF ENERGY IN THE SPECTRUM OF AN ACETYLENE FLAME
}

By W. W. Coblentz

CONTENTS

Page

I. Introductory statement. ............................ $6_{39}$

II. Optical properties of the acetylene flame.................. 640

III. Revision of data previously published . . . . . . . . . . . . . . . . . 643

IV. Effect of this revision upon previous investigations. . . . . . . . . 647

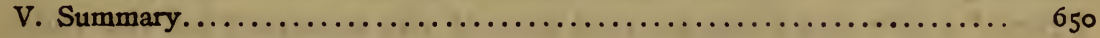

\section{INTRODUCTORY STATEMENT}

The object of this paper is to give some new data on the distribution of energy in the infra-red spectrum of a cylindrical acetylene flame, and to revise some of the previously published spectralenergy data, ${ }^{1}$ pertaining to the violet end of the visible spectrum, which are superseded by the present data.

The data previously published ${ }^{2}$ seem to be the first attempt that has yet been made to supply the demand for quantitative radiometric measurements of spectral-energy distribution. Such data are of value in investigations involving spectrophotometry, color-matching, etc. However, for a source like the acetylene flame, which is weak in intensity in the violet, it is quite impossible to attain high accuracy in the radiation measurements. Nevertheless, in response to requests therefor the data were published, the papers indicating clearly the accuracy attainable and the variability ${ }^{3}$ of the emissivity with thickness of the acetylene flame.

The acetylene flame does not appear to be a suitable standard of spectral radiation, in which high accuracy is desired. As indicated some time ago, the proper procedure is to refer all spectral-energy specifications to a black body at a given temperature, the spectral-energy curve of which can be computed with greater accuracy than it can be observed in the violet end of the spectrum. In recent years great advances have been made in the construction of black bodies; in the establishment of a high

B. S. Bulletin, 13, p. 355; 1916.

B. S. Bulletin, 7 p. 253 , 19r1; 13, p. 355, r9r6.

$145824^{\circ}-20$
3 B. S. Bulletin, 13, p. 357; r9r6.

B. S. Bulletin, 5, p. 349; r909. 
temperature scale; in the determination of the radiation constants; and in the specification of the temperature of sources of high intensity (for example, the gas-filled tungsten lamp), in terms of the color temperature of a black body. Hence, it seems opportune to advocate the specification of spectral energy, colorimetric and other similar specifications in terms of a black body at a given temperature, the spectral-energy curve of which can be computed and checked radiometrically. In this manner experimenters will be using a uniform scale of spectral-energy distribution, whatever the actual energy distribution may be in the spectrum.

As will be discussed more fully on a subsequent page, until recently, when radiometric measurements were supplied to Priest, ${ }^{5}$ there has been no real test of the accuracy of the radiometric measurements or of color matching in terms of these measurements. The energy curve for acetylene as obtained by color matching is high in the violet, but from $0.5 \mu$ to $0.75 \mu$ the two curves are in excellent agreement. This test indicated the possibility of the acetylene-energy data being too high in the violet, as previously surmised. ${ }^{\circ}$

Recently $\mathrm{Hyde}^{7}$ and his collaborators, in an interlaboratory comparison, by color matching a tungsten lamp against the acetylene flame, placed the color temperature of the Eastman Kodak standard burner at $2360^{\circ} \mathrm{K}$. From this test, also, it appears that the directly observed radiometric measurements may be too high in the region of $0.4 \mu$ to $0.48 \mu$. However, as will be shown below, in the spectral region from $0.5 \mu$ to $0.75 \mu$ the observed data are in excellent agreement with those computed on the basis of color temperature.

\section{OPTICAL PROPERTIES OF THE ACETYLENE FLAME}

As a result of the combustion of acetylene gas the emission spectrum of the acetylene flame is a composite of the radiation (I) from incandescent carbon particles, (2) from water vapor and (3) from carbon dioxide. The heated carbon dioxide gives bands of selective emission at $2.7 \mu$ and $4.4 \mu$. The water vapor gives a somewhat continuous spectrum at $\mathrm{I} \mu$ to $6 \mu$ with bands of selective emission in the region of $I .4 \mu$ and $I .85 \mu$. This is well illustrated

S Priest, Phy. Rev. (2), 10, p. 208; x9r7. (See Fig. 3.)

B B. S. Bulletin, 13, p. 359; I9r6.

'Hyde, Forsythe and Cady, Phys. Rev., (2) 13, p. r57' rgrg. 
in the emission spectrum of a bunsen acetylene flame published by Stewart. ${ }^{8}$

The emission spectrum of the incandescent carbon particles is supposed to be smooth and continuous (free from indentations or protuberances), with a maximum of emission at about $\mathrm{I} .28 \mu$ to $\mathrm{I} \cdot 3 \mu$.

Observations of a flat flame, viewed flatwise, $F$, and edgewise, ${ }^{9}$ $E$, also on a cylindrical flame, $C$, Fig. I, do not show a marked difference in the position of the emission maximum. These data were obtained six years ${ }^{10}$ ago but not published. The slope at

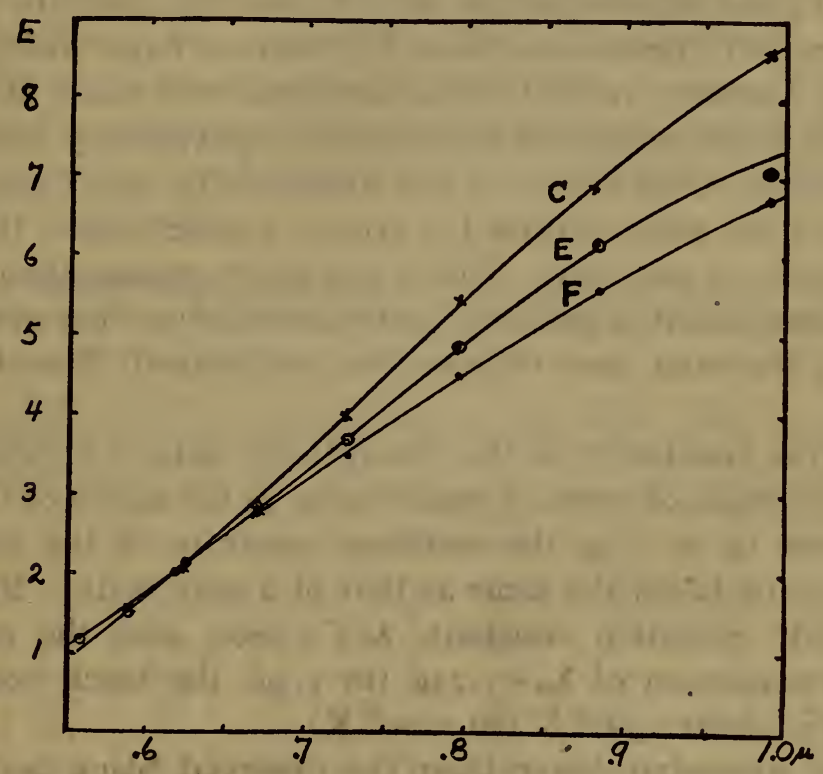

FIG. I.-Spectral energy distribution of acetylene flame: $C=$ cylindrical flame; also flat flame viewed flatwise, $F$, and edgewise, $E$.

$0.5 \mu$ to $0.8 \mu$ of the spectral-energy curve of the cylindrical flame is much steeper than that of the flat flame, indicating a much lower black body color temperature, as observed experimentally.

With the assistance of $\mathrm{H}$. Kahler, recently an examination was made of the emission of the Bray tip (Eastman Kodak Co.burner). The emission maximum was not quite smooth, owing to atmospheric absorption which produced a depression at $I .4 \mu$ in the spectral-energy curve. However, the maximum appears to be at I. $28 \mu$ to $\mathrm{I} .3 \mathrm{O} \mu$, corresponding to a black (gray) body temperature of 2225 to $2250^{\circ} \mathrm{K}$.

${ }^{8}$ Stewart, Phys. Rev., 13, p. 272; rgor.

O B.S. Bulletin, 7, p. 263; rgrr.

10 B. S.Bulletin, 9, p. 98 ; rgra, gives data on the emissivity of different parts of the flame. 
Ångström ${ }^{11}$ found that thin layers of soot, at room temperatures, decrease rapidly in absorption, from the visible to the infrared, thus behaving like a turbid medium. However, incandescent carbon particles appear to have a maximum absorption in the visible spectrum. ${ }^{12}$ Although the discovery of an absorption band may appear novel, the writer was recently informed by Dr. Ångström that some years ago this phenomenon was observed also in his laboratory.

Beyond $i \mu$ the absorptivity of the flame is fairly constant. While no great reliance can be placed upon the absolute value of the absorptivity measurements of a cylindrical flame which is only $3 \mathrm{~mm}$ in diameter, recently measurements were made of the absorptivity of the cylindrical flame used in the visibility work. For wave lengths $0.88 \mu$ and $I .2 \mu$ the absorptivity was found to be practically the same, within the errors of observation, the value being about 2.2 per cent. This is not much greater than that of a flat flame, and it is probably to be expected in view of the fact that only the outer layer of gas of the (cylindrical) flame is incandescent.

From the constancy of the absorptivity data it appears that, within the limits of errors of observation, in the region of the spectrum from $I \mu$ to $I .5 \mu$ the emission spectrum of the acetylene flame may be taken the same as that of a gray body. From the black body radiation constant, $\lambda_{m} T=2890$, and the observed emission maximum of $\lambda_{m}=1.25 \mu$ (to $I .3 \mu$ ) the black body temperature is about $2300^{\circ} \mathrm{K}$ (to $2220^{\circ} \mathrm{K}$ ).

This is somewhat lower than the observed black body color temperature for the visible spectrum, but it is to be expected from consideration of the absorptivity data. For, as we extend our observations toward the violet end of the spectrum, the absorptivity and hence the emissivity becomes higher and higher, and the slope is that of a black body at a higher temperature than obtains for the infra-red energy curve at I. $3 \mu$.

If we multiply the spectral-energy curve of a black body at $2300^{\circ} \mathrm{K}$ by the observed absorptivities, the resulting spectralenergy curve has a slight maximum at $0.65 \mu$, and the slope of the curve in the visible spectrum indicates a temperature higher than $2300^{\circ} \mathrm{K}$, though not in exact agreement with the energy curve for the observed color temperature of $2360^{\circ} \mathrm{K}$. 
The direct radiometric observations do not show a distinct emission maximum in the visible spectrum, though spectrophotometric data are at hand ${ }^{13}$ showing the possibility of such a condition. From the data at hand on emission and absorption it would appear that one should not expect to obtain a smooth spectral-energy curve in the visible spectrum of the acetylene flame, which can be superposed exactly upon the spectral-energy curve of a black body at a temperature corresponding with the color of the flame. The observed spectral-energy data of the acetylene flame given in Table I may, therefore, perhaps be nearer to the true curve than are the computed data. The observed spectralenergy curve of the gas-filled tungsten lamp on normal operation is practically a straight line from $0.5 \mu$ to $0.7 \mu$, though the corresponding energy distribution for a black body color temperature shows some curvature.

TABLE 1.-Spectral Energy Distribution of a Cylindrical Acetylene Flame; $\mathrm{Ob}$ served and Computed Using Wien's eq., $C_{2}=14350$ and $T=2360^{\circ} \mathrm{K}$.

\begin{tabular}{c|c|c|c|c||c|c|c|c|c}
\hline $\begin{array}{c}\text { Wave length } \\
\mu=0.001 \mathrm{~mm}\end{array}$ & $\begin{array}{c}\text { E, ob- } \\
\text { served } \\
1911-1916\end{array}$ & $\begin{array}{c}\mathbf{E}_{1,} \text { re- } \\
\text { vised } \\
1919\end{array}$ & $\begin{array}{c}\mathbf{E}^{\prime}, \text { com- } \\
\text { puted }\end{array}$ & $\begin{array}{c}100 \mathbf{E}^{\prime} \\
\mathbf{E}_{1}\end{array}$ & $\begin{array}{c}\text { Wave length } \\
\mu=0.00 \mathrm{~mm}\end{array}$ & $\begin{array}{c}\text { E, ob- } \\
\text { served } \\
1911-1916\end{array}$ & $\begin{array}{c}\mathbf{E}_{1} \text { re- } \\
\text { vised } \\
1919\end{array}$ & $\begin{array}{c}\mathbf{E}^{\prime}, \text { com- } \\
\text { puted }\end{array}$ & $\frac{100 E^{\prime}}{E_{1}}$ \\
\hline $0.400 \mu$ & 5.9 & 5 & 3.3 & 66 & $0.600^{\mu}$ & 62.1 & 62.5 & 62.9 & 100.6 \\
.425 & 8.2 & 7 & 5.5 & 79 & .620 & 73.0 & 73.3 & 74.0 & 101.1 \\
.440 & 10.0 & 8.5 & 7.6 & 89.4 & .625 & 75.7 & 76.1 & 76.8 & 100.8 \\
.450 & 11.5 & 10.0 & 9.25 & 92.5 & .640 & 84.7 & 85.0 & 86.0 & 101.1 \\
.460 & 13.0 & 11.8 & 11.2 & 94.9 & .650 & 91.1 & 91.2 & 92.1 & 101.0 \\
.475 & 16.0 & 15.0 & 14.6 & 97.4 & .660 & 97.4 & 97.6 & 98.5 & 100.7 \\
.500 & 21.9 & 20.9 & 21.0 & 100.5 & .675 & 107.5 & 107.5 & 108.0 & 100.4 \\
.520 & 27.9 & 27.5 & 27.3 & 99.3 & .680 & 110.9 & 110.9 & 111.3 & 100.7 \\
.525 & 29.5 & 29.2 & 29.2 & 100.0 & .700 & 124.6 & 124.1 & 124.1 & 100.0 \\
.540 & 35.0 & 34.6 & 34.6 & 100.0 & .720 & 138.5 & 137.5 & 137.2 & 99.8 \\
.550 & 38.9 & 38.9 & 38.8 & 99.8 & .725 & 141.9 & 141.0 & 140.5 & 99.6 \\
.560 & 42.9 & 42.9 & 43.1 & 100.4 & .740 & 152.0 & 151.0 & 150.2 & 99.5 \\
.575 & 49.8 & 49.8 & 49.9 & 100.2 & .750 & 158.9 & 157.9 & 157.2 & 99.5 \\
.580 & 52.2 & 52.2 & 52.4 & 100.3 & $a .750$ & 163.7 & 163.0 & 157.2 & 96.5 \\
\hline
\end{tabular}

a Bray tip.

\section{REVISION OF DATA PREVIOUSLY PUBLISHED}

The numerical data, previously published, ${ }^{14}$ on spectral-energy distribution of the acetylene flame, were based upon observations extending from $0.45 \mu$ to $0.75 \mu$ made with a mirror spectrometer and fluorite prism; also with a glass-lens spectrometer and flintglass prism.

13 Nichols and Merritt, Phys. Rev., 30, p. 328; 19ro. See also B. S. Bulletin, 7, p. 268 (Fig. 8), 1911, for ratios of emissivities which indicate such a maximum.

10 B. S. Bulletin, 7, p. 253, I911; 13, p. 355, 1916. 
Hoping to overcome the difficulties in correcting for absorption, a spectrometer consisting of a quartz prism and two planoconvex quartz lenses was used in obtaining the spectral energy in the violet from $0.4 \mu$ to $0.5 \mu$.

In one of the previous papers ${ }^{15}$ an estimate is given of the accuracy attainable in making the observations. It is based upon the deviations of the observations from the mean value, and it is shown that, whereas in the red end of the spectrum an accuracy of 0.5 per cent is attainable (provided the flame stays constant), an accuracy of ro per cent is hardly attainable in the violet where the intensity is low. Unfortunately, no mention is made of the difficulties and uncertainties in reducing these observed data from the prismatic into the normal spectrum. The slit-width factor is probably accurate to I per cent. The greatest uncertainty is the correction for spectral purity, also for absorption in the glass prism, or the silver mirrors, or both, in the violet end of the spectrum. Corrections ${ }^{16}$ must be made also for diffuse light. Furthermore, when using the quartz spectrometer, the lenses not being achromatic, a correction was made for change in aperture with change in focal length for different parts of the spectrum. A recent examination of the original computations shows that the published data have probably been overcorrected (by 5 to ro per cent in the violet) for change in aperture, so the utility of a chromatically uncorrected instrument for making radiometric measurements is questioned. For example, suppose that we have two spectral lines of widely different wave lengths (say red and blue) but of equal energy value as measured with achromatic apparatus. Using the simple quartz lens system, suppose that a red spectral line ro $\mathrm{mm}$ high and $0.5 \mathrm{~mm}$ wide-that is, an image of a spectrometer slit-falls upon a thermopile receiver. It represents a certain amount of energy. Increasing the aperture of the objective telescope by decreasing the focal length (to measure the blue line) increases the energy flux per unit area-that is, energy density-but, the aperture of the collimating lens being unchanged, the total energy falling upon the thermopile remains the same as before.

The problem is not quite so simple as this in practice, seeing that we are dealing with a continuous spectrum. Moreover, in view of possible errors owing to the mechanical difficulties involved

15 B.S. Bulletin, 7, p. 252; 1916.

${ }^{16}$ B.S. Bulletin, 14, p. 229 , 1917 , gives diffuse light tests of the acetylene flame as used in the visibility work. 
in using a nonachromatic spectrometer, the revised data, obtained with the quartz lens spectrometer at $0.4 \mu$ to $0.45 \mu$ can not be given full weight. The revised data are given in Table $I$. They represent a careful reading of the values, after redrawing the curve through the original spectral energy data a copy of which is given in Fig. 3 of the preceding paper on this subject. In many cases the differences between the old and the new readings are caused by lack of exactness in making up the previous table. For wave lengths less than $0.5 \mu$ the present data were obtained from measurements with a flint-glass prism and lens spectrometer; also from a recalculation of the original data obtained with the quartz-lens spectrometer, no correction being made for change in aperture. The uncertainty in the spectral purity factor at $0.4 \mu$ to $0.45 \mu$ amounts to several per cent. But, from $0.46 \mu$ (where the difference between the observed and computed curves amounts to only about 5 per cent) to $0.75 \mu$, these two curves can be considered in exact agreement. At $0.4 \mu$ the observed data may be somewhat too high, owing to diffuse light. However, it will require more than a color-match test as compared with a radiometric match to show that the observed data at $0.4 \mu$ are entirely incorrect. Sometime ago it was shown ${ }^{17}$ that, in two widely different sources of radiation (for example, a tungsten lamp and a Nernst glower) which are set to a color-match, the two spectral-energy curves intersect at an extremely small angle, thus giving the appearance of exact coincidence throughout that part of the spectrum to which the eye is the most sensitive. The measurements being comparative the various correction factors apply to both sources, and a higher accuracy is possible than can be obtained in determining absolute intensities.

Calculations made at the time the data were obtained six years ago, indicated that, for the region of $0.45 \mu$ to $0.75 \mu$ the spectralenergy distribution of the acetylene flame was that of a black body at about $2100^{\circ} \mathrm{C}\left(2370^{\circ} \mathrm{K}\right)$ though, at the time, no significance was attached to it.

Recently, from an interlaboratory color-match comparison of a tungsten lamp with the acetylene flames used in this laboratory and in the Eastman Kodak Laboratory, Hyde ${ }^{18}$ obtained a coior temperature of $2360^{\circ} \mathrm{K}$ for the Eastman Kodak type of lamp. He obtained a somewhat higher temperature for the burner used

17 B. S. Bulletin, 7, p. 29I (Fig. 13), rgro.

${ }^{28}$ Hyde, Forsythe and Cady, Phys. Rev. (2), 13, p. I57; rgrg. 
by the writer. However, this was for a slit $10 \mathrm{~cm}$ in height instead of an $8 \mathrm{~cm}$ slit which was used in the spectral-energy measurements for the visibility data. In view of the fact that, within experimental errors of observation, the two types of burners had the same spectral-energy curves, their black body color temperatures may be taken at $2360^{\circ} \mathrm{K}$.

In Table I, column 2 gives the spectral-energy data previously published, and column 3 gives the revised data. Column 4 gives the spectral-energy distribution of a black body at $2360^{\circ} \mathrm{K}$, kindly computed by $\mathrm{H}$. Kahler and carefully superposed upon the observed data, using a scale which was sufficiently large to eliminate errors (estimated to be 0.5 per cent) in drawing the curves and reading off the data. Column 5 gives the ratios of the revised acetylene energy data to the computed data for $2360^{\circ} \mathrm{K}$. If the two curves coincided exactly, these ratios would be 100 . As shown in the table, between $0.48 \mu$ and $0.75 \mu$ the departure from exact coincidence is only from $\pm I$ to 2 per cent, which is within the experimental errors involved in observing the data and reducing them to a normal spectrum. But considering the data previously published, given in column 2 of Table $I$, the greatest departure from coincidence of the observed and the computed curves in the spectral region of $0.5 \mu$ to $0.75 \mu$ is only from \pm 2 to \pm 4 per cent, instead of 8 per cent as stated by Hyde.

The color temperature data of Hyde, therefore, confirm the radiometric measurements in the region of $0.48 \mu$ to $0.75 \mu$. This is the region in which the radiometric measurements are of importance in the writer's work on the visibility of radiation, and in which it was possible to make precise radiometric measurements.

Although the writer doubts the reliability of the color-match test in the extreme violet of the spectrum, where the eye is very insensitive and subject to great decrease in sensitivity with age, he concurs in the recommendation made by Hyde that the spectral-energy distribution of the acetylene flame (using a certain type of burner) in the visible spectrum is satisfactorily represented by the black body curve at $2360^{\circ} \mathrm{K}$, as given in Table $\mathrm{I}$. A difference of $10^{\circ}$ or even $50^{\circ} \mathrm{K}$ is hardly to be considered in view of the great divergence in burners, the effect of humidity, etc. Moreover, the color temperature varies greatly for different parts of the flame ${ }^{10}$-for example, $2368^{\circ} \mathrm{K}$ for the whole flame and $2448^{\circ} \mathrm{K}$ for one spot. 


\section{EFFECT OF THIS REVISION UPON PREVIOUS INVESTIGATIONS}

As already stated, in order to test the accuracy of his new method of color matching, Priest ${ }^{20}$ compared the writer's radiometrically determined spectral-energy curve of a 500-watt gasfilled tungsten lamp with that of the acetylene flame as modified by the quartz-nicol system used in making the color match. The curve for the acetylene flame is too high in the violet; but from $0.5 \mu$ to $0.75 \mu$ the two spectral-energy curves are in exact coincidence. Using the revised spectral-energy data of Table I, brings them into a close agreement also in the region of $0.45 \mu$ to $0.48 \mu$.

Some doubt has been expressed as to the accuracy of measuring the energy at the observing slit, in visibility work, instead of computing it from the color temperature of the source. In the latter case it is necessary to reduce the data to the prismatic distribution for the particular apparatus used; and there is no check on the actual energy distribution at the slit.

On the other hand, to measure the prismatic spectral energy distribution at the observing slit, reduce it to a normal spectrum, compute the temperature of the source and find it in agreement with the directly observed color temperature, as has just been found, constitutes a real test of the accuracy attained in the energy evaluation. It would be more logical to doubt the computed spectral energy distribution which has not been checked by direct radiometric observations.

As already stated, instead of a disagreement between the color temperature test and the spectral radiation measurements, the independent check by Hyde and his collaborators confirms the direct radiometric observations on the spectral-energy distribution of the acetylene flame, in the region of $0.48 \mu$ to $0.75 \mu$, as used in the visibility of radiation work, and leaves the visibility data ${ }^{21}$ unchanged in this part of the spectrum. If any correction is to be made it falls at $0.60 \mu$ to $0.67 \mu$ (see Table I), being a decrease of 0.5 to I per cent in the visibility data.

As for the visibility data in the violet, they were obtained separately from the main part of the curve (from $0.5 \mu$ to $0.7 \mu$ ) using a much lower field illumination, which may account for the higher values as compared with other experimenters. In view of the great variability of visibility in the violet, especially with age,

${ }^{20}$ Priest, Phys. Rev. (2) 10, p. 208; 1917. (See Fig. 3.)

21 B.S. Bulletin, 14, p. 168; 1917. 
one can hardly establish an "average visibility curve" and hence no attempt is made to revise the data (in the violet) previously published. If the data on 125 observers previously published serve no other purpose, they are useful in demonstrating the extraordinary varability in sensibility of the eye, ${ }^{22}$ and hence the futility of attempting to establish an "average eye without arbitrarily choosing the observers."

In a recent paper Ives ${ }^{23}$ expresses the opinion that, in the writer's visibility work, the field brightness should have been somewhat higher $(200 \mathrm{~m}$. c.), in order to meet the requirements in photometry; and that, as a consequence of this low illumination, the visibility curve is shifted too far toward the red. It is therefore relevant to say, in reply to this criticism, that a field brightness of $50 \mathrm{~m}$. c. was used on the sectored disk, because it permitted observing over a wide range of the spectrum, and because the visibility curve for this illumination was found in agreement ${ }^{24}$ with that obtained for an illumination of 350 to $780 \mathrm{~m}$. c., as was previously observed by Nutting and others. At the time the visibility work was undertaken, Ives ${ }^{25}$ specified an illumination of $25 \mathrm{~m}$. c. on the disk, for flicker photometry, but no specifications were given for obtaining the visibility curve. His visibility ${ }^{26}$ curves for $68 \mathrm{~m}$. c. and $270 \mathrm{~m}$. c. illumination on the disk appeared to be sufficiently close in agreement to conclude that the lower illumination was satisfactory, as was proved by the subsequent experiments just cited. However, it is conceivable that the average visibility curve of a large group of observers, whose eyes have not been subjected to years of hard usage, would be different for these same illuminations.

In the paper on visbility (loc. cit.) attention was called to the great number of red-sensitive subjects, whose observations shift the average visibility curve toward the red to a greater extent than would obtain if a smaller number of red-sensitive subjects had been used. The alleged shift of the visibility curve toward the red is no doubt owing in part to the relatively large number of red-sensitive subjects and the relatively low number of greensensitive subjects found in the I 25 persons tested by the writer, ${ }^{27}$ rather than attributing it entirely to a low field illumination. On

\footnotetext{
22 B.S. Bulletin, 14, p. 206; 1917 . (See the composite curve, Fig. 13.)

${ }^{23}$ Ives, Jour. Franklin Inst., 188, p. 227 ; 1919.

24 See Fig. 2, B. S. Bulletin, 14, p. 178 ; 1917.

${ }^{25}$ Ives, Trans. Illum. Eng. Soc., 10, p. $317 ;$ rgrs.

${ }^{26}$ Ives, Phil. Mag. (6), 24, pp. I49, 352, 744, 845, 853; r9r2.

${ }^{27}$ See Table 3, B. S. Bulletin, 14, p. 205; r917.
} 
the other hand in the group of 2 I observers examined by Nutting, ${ }^{28}$ there were as many green-sensitive as average subjects (4 of each group, see Table 3 loc. cit.), and there was one unusually bluesensitive subject. These five subjects constitute almost one-fourth of the total number examined, and their observations tend to suppress the average visibility curve in the red. The average visibility curve of Nutting, which happens to meet Ives's requirements, is not shifted quite so far to the red as is the average visibility curve obtained by the writer. ${ }^{29}$

Nutting used an illumination of $350 \mathrm{~m}$. c. on the sectored disk; but did not use an illuminated surrounding field as was done by the writer and as specified by Ives. As originally published, the visibility curve obtained by Nutting was in error owing to improper energy evaluation of his Bray tip, acetylene flame. Subsequently the spectral-energy distribution of this burner was determined by the writer with the same spectrometer used in his visibility work and found in agreement with the herein described data. Using these spectral energy measurements, the corrected visibility curve of Nutting ${ }^{30}$ is in close agreement with that obtained by the writer, except in the red end of the spectrum, where it is slightly lower, and happens to be in agreement with tests made on a physical photometer. ${ }^{31}$

Neither Nutting nor the writer attempted to obtain their visibility data for normal pupil illumination; though, by using $35^{\circ}$ m. c. on the disk, the former had practically an artificial pupil illumination corresponding to $25 \mathrm{~m}$. c. normal pupil as previously used by Ives. But this illumination ( $350 \mathrm{~m}$. c. on the disk) was constant for all observers, whereas Ives found that the artificial pupil illumination varied greatly for various observers, the highest illumination on the disk being twice the lowest. Ives's criticism that the "curves are not coordinated with any accurately specified observing conditions nor method of choosing observers" applies to Nutting's as well as the writer's visibility curves, and hence, aside from the higher illumination used by Nutting, there is no reason for selecting either one simply because it happens to fit the tests of a physical photometer.

Evidently, where great refinement is required, the visibility curve of the so-called average eye should be based upon observations made by a selected group of observers, having closely normal

${ }^{28}$ Nutting, Trans. Illum. Eng. Soc., 9, p. 663; 1914.

${ }^{29}$ See Fig. I4, B. S. Bulletin, 14, p. 216 ; 1917.
${ }^{30}$ B. S. Bulletin, loc. cit., Fig. 14.

${ }^{31}$ Ives, Jour. Franklin Inst., 188, p. 222; rgrg. 
color vision. Fortunately the writer's visibility data are classified as to normality of visibility ${ }^{32}$ and the average value of the visibility curve of observers having closely normal color vision is easily obtained. The average visibility data of 29 observers, classified as normal in the previous paper, are given in column 3 of Table 2, along with the previously published data (column 2) on I 25 observers. The total number of observers being so large, the effect of the observers having abnormal color vision is quite inappreciable upon the average visibility curve, except in the extreme red, as just mentioned.

TABLE 2.-Visibility of Radiation of a Group of 125 Observers and of Selected Group of 29 Observers Having Closely the Average Visibility

\begin{tabular}{|c|c|c|c|c|c|}
\hline \multirow{2}{*}{$\begin{array}{l}\text { Wave length } \\
\mu=0.001 \mathrm{~mm}\end{array}$} & \multicolumn{2}{|c|}{ Visibility of radiation } & \multirow{2}{*}{$\begin{array}{l}\text { Wave length } \\
\mu=0.001 \mathrm{~mm}\end{array}$} & \multicolumn{2}{|c|}{ Visibility of radiation } \\
\hline & 125 observers & 29 observers & & 125 observers & 29 observers \\
\hline $0.493 \mu$ & 0.223 & 0.230 & $0.596 \mu$ & 0.734 & 0.735 \\
\hline .502 & .350 & .357 & .604 & .636 & .636 \\
\hline .512 & .553 & .559 & .613 & .517 & .517 \\
\hline .523 & .771 & .778 & .623 & .390 & .387 \\
\hline .534 & .908 & .908 & .633 & .267 & .264 \\
\hline .546 & .983 & .987 & .643 & .165 & .164 \\
\hline .552 & .998 & 1.000 & .654 & .095 & .093 \\
\hline .559 & 1.000 & .998 & .665 & .046 & .045 \\
\hline .573 & .952 & .955 & .678 & .0202 & .0197 \\
\hline .580 & .899 & .900 & .690 & .0086 & .0075 \\
\hline .587 & .823 & .824 & .703 & .0034 & .0033 \\
\hline
\end{tabular}

\section{SUMMARY}

The object of this paper is to give some new data on the distribution of energy in the infra-red spectrum of a flat and of a cylindrical acetylene flame. The paper gives, also, a revision of previously published spectral-energy data pertaining to the violet end of the visible spectrum.

The optical properties of the flame are discussed, and it is shown that, owing to the high selective absorption of the flame in the visible spectrum, the apparent color temperature is higher than that obtained from a consideration of the maximum emission in the infra-red.

The radiometric, as well as the color-temperature measurements, indicate that in the visible spectrum, from $0.48 \mu$ to $0.74 \mu$ the spec- 
tral-energy distribution of the central zone of the cylindrical acetylene flame is that of a black body at $2360^{\circ} \mathrm{K}$.

It is shown that the revision of the spectral-energy data of the acetylene flame has no effect upon the previously published visibility data in the region of the spectrum from $0.48 \mu$ to $0.75 \mu$. This is the part of the spectrum in which an attempt had been made to attain high accuracy in the visibility data. A table is given of the visibility of the average of 29 . observers having closely normal color vision, as observed with a flicker photometer.

Washington, September 3, I9I9.

Nore.-The criticism recently made by $\mathrm{Hyde}^{33}$ and his collaborators that the writer's data (particularly in the red) do not fit the black body energy distribution better than 7 to 8 per cent is inconsistent with their own observations. Using a spectral pyrometer, they find that at $0.75^{\mu}$ the observed emissivity is slightly (about 7 per cent) higher than that of a black body at $2360^{\circ} \mathrm{K}$.

In other words, their data show, as was found by the writer ${ }^{34}$ some years ago, that, in making a color match, the eye is not able to distinguish a difference in the spectral energy match amounting to perhaps 3 to 5 per cent in the extreme red end $(0.75 \mu)$ of the visible spectrum. The supposed disagreement appears to be in our interpretation of the data, rather than in the experimental results themselves. For, if the emissivity is higher in the red, then the ratios must decrease as shown in column ro of Table r.

WASHINGTON, January 9, Ig2o.

33 Hyde, Forsythe and Cady. Phys. Rev. (2), 14, p. 382; 1919.

3 B. S. Bulletin, 6, p. 317; rgro. 